\title{
Acceso a información científica libre sobre la enfermedad por coronavirus 2019 (COVID-19) en Pubmed y Tripdatabase Pro
}

\section{Access to free scientific information on coronavirus disease 2019 (COVID-19) in Pubmed and Tripdatabase Pro}

\author{
Shamir Barros-Sevillano ${ }^{1,2, a}$, Dianilet Delgado-Gasco ${ }^{1, b}$, Diego Vásquez-Vértiz ${ }^{1, c}$, \\ Joshuan Barboza ${ }^{1,2, d}$ \\ ${ }^{1}$ Escuela de Medicina, Universidad César Vallejo. Trujillo, Perú. \\ ${ }^{2}$ Tau-RELAPED Group. Trujillo. Perú. \\ a Estudiante de medicina, Sociedad Cientifica de Estudiantes de Medicina de la Universidad César Vallejo. ORCID: https://orcid.org/0000-0003-2997-2092 \\ b Estudiante de medicina, Sociedad Cientifica de Estudiantes de Medicina de la Universidad César Vallejo. ORCID: https://orcid.org/0000-0001-7760-4265 \\ ${ }^{c}$ Estudiante de medicina, Sociedad Cientifica de Estudiantes de Medicina de la Universidad César Vallejo. ORCID: https://orcid.org/0000-0002-1009-7569 \\ ${ }^{d}$ Magíster en ciencias de la investigación clínica. ORCID: https://orcid.org/0000-0002-2896-1407
}

An Fac med. 2020;81(2):256-9. / DOI: https://doi.org/10.15381/anales.v81i2.17802

Correspondencia:

Shamir Barros Sevillano

shamir.bs17@gmail.com

Recibido: 16 de mayo 2020

Aprobado: 14 de junio 2020

Publicación en línea: 30 de junio 2020

Conflictos de interés: Los autores declaran no tener conflictos de interés.

Fuente de financiamiento:

Autofinanciado

Citar como: Barros-Sevillano S,

Delgado-Gasco D, Vásquez-Vértiz D, Barboza J. Acceso a la información cientifica libre sobre la enfermedad por coronavirus 2019 (COVID-19) en Pubmed y Tripdatabase pro. An Fac med. 2020;81(2):256-9.DOI: https://doi. org/10.15381/anales.v81i2.17802
Sr. Editor,

La enfermedad por coronavirus 2019 (COVID-19) producida por el reciente coronavirus del síndrome respiratorio agudo severo 2 (SARS-CoV-2), es catalogada como una emergencia de salud pública por sexta vez en la historia por la Organización Mundial de la Salud (OMS) (1). Al 22 de junio de 2020 existen 8708008 casos confirmados de COVID-19 entre 188 países/ regiones, con un número de 461715 muertes, convirtiéndose en una de las pandemias más complejas por su rápida propagación a nivel mundial ${ }^{(2)}$.

La relevancia del acceso libre a información científica en estos momentos es primordial para la toma de decisiones, pero las barreras de pago siempre han afectado la capacidad de los investigadores para acceder a la información, planteando enfoques con fines de lucro y exacerbando las desigualdades en los recursos académicos entre el norte global y el sur global ${ }^{(3)}$. Sin embargo, ante el panorama reciente, no solo las revistas académicas han ajustado sus modelos de publicación, acceso y distribución, sino que también los repositorios en línea, motores de búsqueda y bases de datos de indexación han tenido que actualizar sus servicios para impulsar una mejor accesibilidad y difusión de contenido relacionado con el nuevo coronavirus.

Presentamos los resultados de una investigación bibliométrica, realizada en el mes de mayo 2020, que tuvo como objetivo brindar una visión general sobre las publicaciones y plataformas en línea disponibles para el acceso a información científica libre sobre la COVID-19 encontradas en Pubmed y Tripdatabase Pro, utilizando estrategias de búsqueda bibliográfica con los descriptores MeSH ("COVID-19" OR "2019 novel coronavirus infection" OR "CO- 
VID19" OR "coronavirus disease 2019" OR "coronavirus disease-19" OR "2019nCoV disease" OR "2019 novel coronavirus disease" OR "2019-nCoV infection").

Se encontraron 10670 resúmenes como acceso libre asociados a la estrategia de búsqueda bibliográfica en Pubmed y 1383 resúmenes en Tripdatabase Pro. Se eliminaron 768 resúmenes duplicados, obteniéndose un total de 11285 resúmenes. Todo este material virtual que permite ser usado con cualquier propósito legal ${ }^{(4)}$, otorga un impacto positivo en estos tiempos de incertidumbre permitiendo que se construya nuevas investigaciones en base a ese conocimiento. La información divulgada principalmente en revistas revisadas por pares, es vital para los tomadores de decisiones durante la emergencia sanitaria ${ }^{(5,6)}$; por ejemplo, la liberación de secuencias completas del genoma viral a través de una plataforma de acceso público y los protocolos de ensayo de reacción en cadena de la polimerasa, permitió diagnosticar con precisión las infecciones al inicio de la emergencia actual.

Del total de artículos encontrados en Tripdatabase Pro, el 43,3\% fueron sinopsis basadas en evidencia, el 26,1\% guías de práctica clínica, el 23\% investigación primaria y un 7,6\% que representa otro tipo de estudios; esto evidencia una variedad de información acerca del COVID-19 y una respuesta eficiente por parte de la comunidad científica. No obstante, los esfuerzos para la notificación acelerada de datos y resultados no debían limitarse a estos, sino incluir estudios de observación, investigación operativa, vigilancia de rutina e información sobre el virus y monitoreo de los programas de control de enfermedades para contrastar el efecto de la enfermedad de una manera integrada.

En el análisis de redes con el software VOSviewer v.16 para identificar la asociación de las palabras clave relacionadas a COVID-19 ("Covid"), las que presentaron mayor asociación fueron: "retrospective studies", "practice guidelines" y "clinical protocols" (Figura 1), lo que podría servir para mejorar las estrategias de búsqueda bibliográfica para estos tipos de eviden- cia científica, ya que las palabras clave, en parte resuelven el problema de autores, investigadores y bibliotecarios que pueden usar diferentes términos para el mismo concepto ${ }^{(7)}$.

Al buscar la fuente de los resultados encontrados como acceso libre en la búsqueda bibliográfica, se logró catalogarlos como sociedad/agrupación, revista, biblioteca, editorial y repositorio cuya información sobre COVID-19 se encontraba como acceso libre, reportándolas con cada una de las especificaciones (Tabla 1). Todo esto impulsa el intercambio de información en tiempo real, favorecida por la implementación de revisiones aceleradas de manuscritos relacionados a COVID-19, tal como como el boletín de la OMS que implementa un protocolo de intercambio de datos "COVID-19 Open". Cuando se envía al boletín, los manuscritos de investigación relevantes para la emergencia del coronavirus se les asignan un identificador de objetos digitales (DOI) y se publican en línea en la colección "COVID-19 Open" mientras se someten a una revisión por pares.

Los servidores previos a la publicación, podrían ayudar aún más a los investigadores para cargar sus documentos antes de enviarlos a una revista revisada por pares, considerando que el Comité Internacional de Editores de Revistas Médicas confirmó que la difusión previa no perjudicaría la publicación de revistas en el contexto de la emergencia de salud ${ }^{(8)}$.

Finalmente, sigamos aprendiendo sobre la deficiencia en el intercambio de datos producidas durante el brote del virus del Ébola en 2013 al $2016{ }^{\text {(9), que }}$ llevó a un acuerdo sobre la necesidad de un intercambio abierto de datos y resultados, especialmente en emergencias sanitarias (10); sin embargo, es necesario continuar los esfuerzos para una divulga-

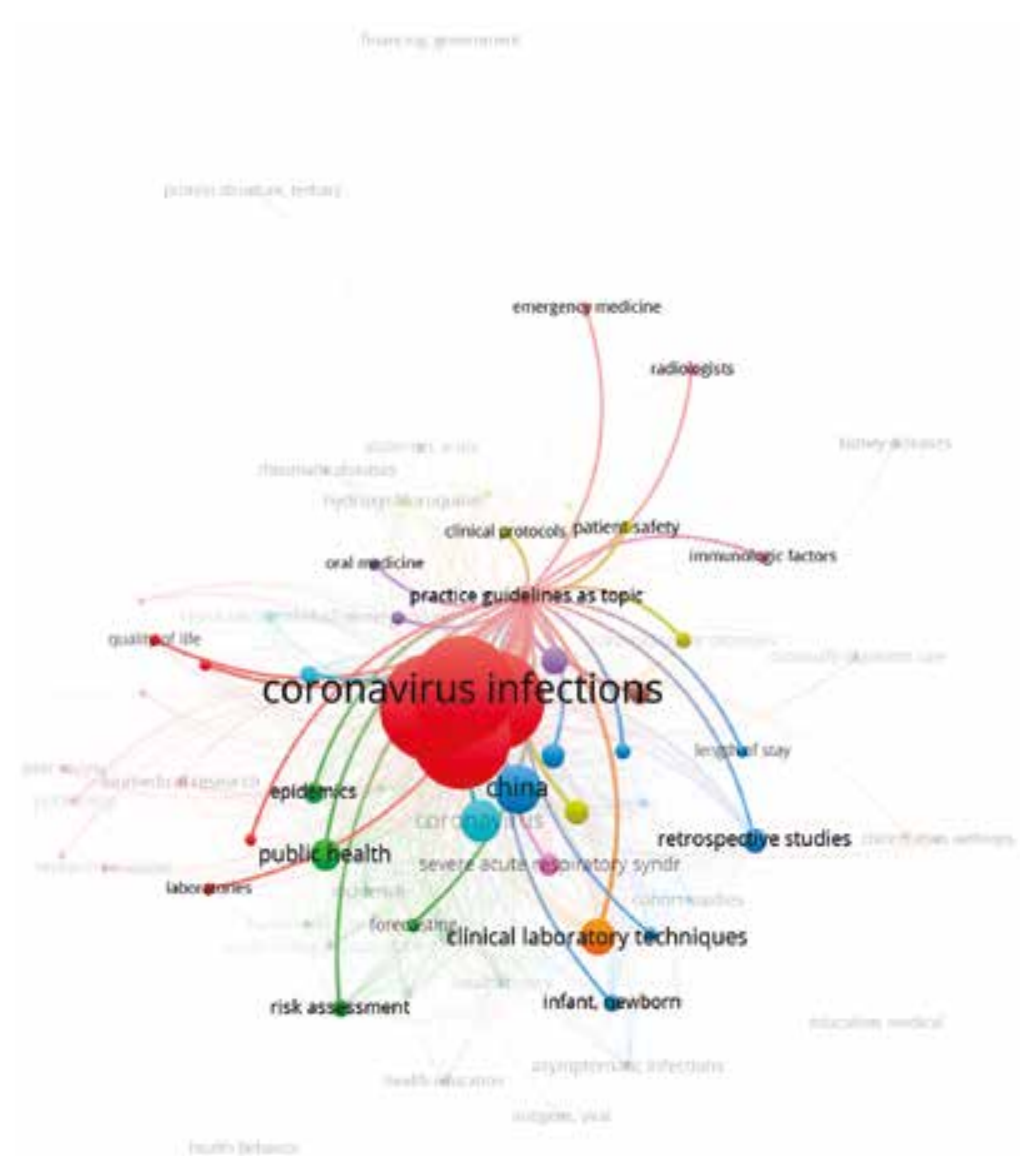

Figura 1. Análisis en red de las palabras clave presentes en los artículos de acceso libre asociadas a COVID-19 "Covid", en Pubmed y Tripdatabase Pro. 
Tabla 1. Fuentes de información de acceso gratuito sobre la enfermedad por coronavirus (COVID-19) en Pubmed y Tripdatabase Pro.

\begin{tabular}{|c|c|c|c|}
\hline Fuente de procedencia & \multirow{2}{*}{ País de origen } & \multirow{2}{*}{$\begin{array}{c}\text { Año de } \\
\text { inicio }\end{array}$} & \multirow{2}{*}{ Usualmente versión - acceso** } \\
\hline Sociedad / agrupación & & & \\
\hline American Chemical Society (ACS) & Estados Unidos & 1876 & Impresa y en línea. Acceso libre a texto completo. \\
\hline Organización mundial de la salud (OMS) & Ginebra & 1948 & Impresa y en línea. Acceso libre a texto completo. \\
\hline European Centre for Disease Prevention and Control (ECDPC) & Suecia & 2005 & En línea. Acceso libre a texto completo. \\
\hline Centers for Disease Control and Prevention & Estados Unidos & 1946 & Impresa y en línea. Acceso libre a texto completo. \\
\hline Canada Foundation for Innovation & Canadá & 1997 & En línea. \\
\hline Chinese Centre for Disease Control and Prevention & China & 1998 & En línea. \\
\hline Sociedad Americana de Microbiología (ASM) & Estados Unidos & 1899 & En línea. \\
\hline American Society of Clinical Oncology & Estados Unidos & 1964 & Impresa y en línea. \\
\hline European Respiratory Society & Suiza & 1990 & Impresa y en línea. \\
\hline American Physical Society & Estados Unidos & 1899 & Impresa y en línea. \\
\hline Global Virus Network & Estados Unidos & 2011 & Impresa y en línea. \\
\hline American Society of Nephrology & Estados Unidos & 1966 & Impresa y en línea. \\
\hline \multicolumn{4}{|l|}{ Revistas* } \\
\hline British Medical Journal (The BMJ) & Reino Unido & 1840 & En línea. \\
\hline Journal of the American Medical Association (JAMA) & Estados Unidos & 1883 & Impresa y en línea. \\
\hline The Lancet & Britania & 1823 & Impresa y en línea. \\
\hline The New England Journal of Medicine (NEJM) & Estados Unidos & 1812 & Impresa y en línea. \\
\hline Science Magazine & Estados Unidos & 1848 & En línea. \\
\hline \multicolumn{4}{|l|}{ Bibliotecas } \\
\hline Public Library of Science (PLOS) & Estados Unidos & 2001 & En línea. Acceso libre a texto completo. \\
\hline Znanium & Rusia & 2011 & En línea. \\
\hline Cochrane & Britania & 1997 & En línea. Acceso libre a texto completo. \\
\hline \multicolumn{4}{|l|}{ Editorial } \\
\hline Elsevier & Países Bajos & 1880 & Impresa y en línea. \\
\hline Oxford University Press & Reino unido & 1586 & Impresa y en línea. \\
\hline Cambridge University Press & Inglaterra & 1534 & Impresa y en línea. \\
\hline Bioone & Estados Unidos & 1999 & Impresa y en línea. \\
\hline Springer Nature & Alemania & 1842 & Impresa y en línea. \\
\hline Annual Reviews & Estados Unidos & 1932 & En línea. \\
\hline Emerald & Reino Unido & 1967 & Impresa y en línea. \\
\hline SAGE Publishing & Estados Unidos & 1965 & Impresa y en línea. \\
\hline Taylor \& Francis & Inglaterra & 1936 & Impresa y en línea. \\
\hline Wiley & Estados Unidos & 1999 & Impresa y en línea. \\
\hline Wolters Kluwer & Países Bajos & 2007 & Impresa y en línea. \\
\hline Brill & Países Bajos & 1683 & Impresa y en línea. \\
\hline Bloomsbury Publishing & Inglaterra & 1986 & Impresa y en línea. \\
\hline \multicolumn{4}{|l|}{ Repositorio } \\
\hline bioRxiv & Estados Unidos & 2013 & En línea. Acceso libre a texto completo. \\
\hline
\end{tabular}

* Solo se consideró las más importantes

**Tipo de acceso a su contenido en condiciones normales.

ción más eficiente, ya que el acceso a texto completo aumenta la transparencia, beneficiando tanto a los médicos como a los pacientes al encontrar evidencia actualizada y completa para mejorar las decisiones. Asimismo, en el marco del COVID-19, compartir la información científica podría ser una forma efectiva de reducir el pánico, ansiedad y mitos entre la sociedad y la comunidad de lectores.

\section{REFERENCIAS BIBLIOGRÁFICAS}

1. World Health Organization Europe [Internet]. 2019-nCoV outbreak is an emergency of international concern. Dinamarca: World Health Organization Europe; 31 de enero 2020 [Fecha de acceso: 3 de junio 2020]. Disponible en: http://www.euro.who.int/en/health-topics/healthemergencies/international-health-regulations/ news/news/2020/2/2019-ncov-outbreak-is-anemergency-of-international-concern

2. World Health Organization [Internet]. Coronavirus disease 2019 (COVID-19) Situation Report - 153.
Ginebra: World Health Organization; 22 de junio 2020 [Fecha de acceso: 22 de junio 2020]. Disponible en: https://www.who.int/docs/default-source/ coronaviruse/situation-reports/20200621-covid19-sitrep-153.pdf?sfvrsn=c896464d_2

3. Tennant JP, Waldner F, Jacques DC, Masuzzo $\mathrm{P}$, Collister LB, Hartgerink CHJ. The academic, economic and societal impacts of Open Access: an evidence-based review. F1000Res. 2016;5:632. DOI: 10.12688/f1000research.8460.3

4. Caballero CV, Marenco Robles R, Heidy MA, Monroy Roja S, Palencia Sánchez D, Rodríguez Torres S. The importance of the Open Access in the bio- 
medical and scientific investigation. Rev Colomb Reumatol. 2008;15(2):93-101.

5. Dye C, Bartolomeos K, Moorthy V, Kieny M. Data sharing in public health emergencies: A call to researchers. Bulletin of the World Health Organization. 2016;94. DOI: 10.2471/BLT.16.170860

6. Whitty CJM, Mundel T, Farrar J, Heymann DL, Davies SC, Walport MJ. Providing incentives to share data early in health emergencies: the role of journal editors. The Lancet. 2015;386(10006):1797-8. DOI: 10.1016/S0140-6736(15)00758-8
7. Moncada-Hernández SG. Cómo realizar una búsqueda de información eficiente: Foco en estudiantes, profesores e investigadores en el área educativa. Investigación educ. 2014;3(10):106-15 DOI: 10.1016/S2007-5057(14)72734-6

8. International Committee of Medical Journal Editors [Internet]. Recommendations for the conduct, reporting, editing, and publication of scholarly work in medical journals. Filadelfia: International Committee of Medical Journal Editors; Diciembre 2020 [citado 2 de junio 2020]. Disponible en: http://www.icmje. org/icmje-recommendations.pdf

9. Coltart CEM, Lindsey B, Ghinai I, Johnson AM, Heymann DL. The Ebola outbreak, 2013-2016: old lessons for new epidemics. Philos Trans R Soc Lond B Biol Sci. 2017;372(1721). DOI: 10.1098/ rstb.2016.0297

10. Modjarrad K, Moorthy VS, Millett P, Gsell P-S, Roth C, Kieny M-P. Developing Global Norms for Sharing Data and Results during Public Health Emergencies. PLoS Med. 2016;13(1):e1001935. DOI: 10.1371/ journal.pmed.1001935 